\title{
QoE Enhancement of Audio-Video IP Transmission with IEEE 802.11e EDCA in Mobile Ad Hoc Networks
}

\author{
Toshiro Nunome and Shuji Tasaka \\ Department of Computer Science and Engineering, Graduate School of Engineering, \\ Nagoya Institute of Technology, Nagoya 466-8555, Japan \\ E-mail: \{nunome, tasaka\}@ nitech.ac.jp
}

\begin{abstract}
This paper compares QoE (Quality of Experience) of audio-video IP transmission with IEEE 802.11e EDCA and that with DCF in mobile ad hoc networks. We also assess QoE of the transmission with TXOP-bursting, which is an optional function of EDCA. QoE assessment of audio-video IP transmission in mobile ad hoc networks involves difficult issues. The wireless nodes can move, and then channel condition changes dynamically. The routing paths may also change as the wireless nodes move. In this paper, we tackle the difficulty of the assessment by means of a continuous time QoE assessment method, which utilizes the method of successive categories continuously in time. For the assessment, at first, we performed computer simulation, where a mesh topology network with mobile nodes is assumed for transmitting audio, video, and data traffic. We then carried out subjective experiment. The assessment results show that EDCA and TXOP enhance QoE of audio-video transmission over mobile ad hoc networks.
\end{abstract}

\section{INTRODUCTION}

New generation wireless networks are expected to provide a wide variety of services with high perceived QoS (Quality of Service) for users. The perceived QoS is referred to as Quality of Experience $(Q o E)$ in ITU-T [1]. It is the most important among QoS's at all levels since the users are the ultimate recipients of the services.

Audio-video IP transmission forms an indispensable basis of networked multimedia applications even in mobile ad hoc networks (MANETs). Enhancement of the output quality of audio-video streams is one of the most important issues.

The IEEE 802.11e EDCA (Enhanced Distributed Channel Access) is an enhanced version of the DCF (Distributed Coordination Function) and can support relative priority services for multimedia transmission [2]. Audio, video, and data traffic have different requirement for QoS from each other. QoS differentiation plays a key role in providing QoE costefficiently in a wireless mobile environment.

A variety of studies on QoS control with the IEEE 802.11e EDCA in ad hoc networks have been reported [3]-[10]. References [3] and [4] propose cross-layer design architectures with the network and MAC layers. Lee et al. employ crosslayer design with the physical and MAC layers [5]. Dynamic priority assignment schemes are proposed in [6], [7] and [8]. Zhu et al. propose a scheme to control contention window $(C W)$ size dynamically according to the traffic condition [9]. Calafate et al. employ a probe-based admission control [10].

Most of the studies discussed above assess only end-toend-level and lower-level QoS for their performance evalu- ation. As mentioned earlier, QoE is the most important QoS for the users. However, none of the studies assess QoE.

QoE of audio-video transmission over infrastructure networks with the IEEE 802.11e EDCA is assessed in [11]. The structure and traffic characteristics of the infrastructure networks are largely different from those of the ad hoc networks. Thus, the effect of EDCA in the ad hoc networks on QoE can differ from that in the infrastructure networks.

In [12] and [13], the authors have also assessed QoE of audio-video transmission over ad hoc networks without mobility. In order to achieve high QoE, the authors have employed link quality-based routing, which takes account of link quality such as signal strength and link utilization level for route selection. However, these references do not consider mobile nodes, which is one of the most important characteristics in mobile ad hoc networks. In addition, it does not assume service differentiation in the MAC layer; the audio, video, and data traffic are transmitted with the same priority.

In this paper, we assess the QoE of audio-video IP transmission with the IEEE 802.11e EDCA over mobile ad hoc networks. As in [13], we employ a continuous time QoE assessment method, which is proposed in [14] and utilizes the method of successive categories continuously in time.

In this study, as a first step, we assume grid topology networks with mobile nodes. It is a simple example of mobile ad hoc networks.

The rest of this paper is organized as follows. Section II summarizes the IEEE 802.11e EDCA. Section III illustrates a methodology of the simulation, and Section IV describes the assessment method of QoE. The assessment results are presented and discussed in Section V.

\section{IEEE $802.11 \mathrm{E}$ EDCA}

The IEEE 802.11e EDCA introduces four ACs (Access Categories) to support differentiated channel access for applications with QoS requirements [2]. A station which supports the EDCA has an individual source buffer for each of the four $\mathrm{ACs}$, and the channel access function based on the CSMA/CA is independently carried out per AC.

In addition, the IEEE 802.11e MAC defines a TXOP (Transmission Opportunity) as an interval of time when a specified station has right to initiate sequences of frame exchange onto the wireless medium. In the EDCA, a station can transmit multiple MAC frames during a TXOP from the same source buffer (AC) until the time reaches the TXOPLimit $[A C]$ [2]. We refer to this scheme as the TXOP-bursting in this paper. 


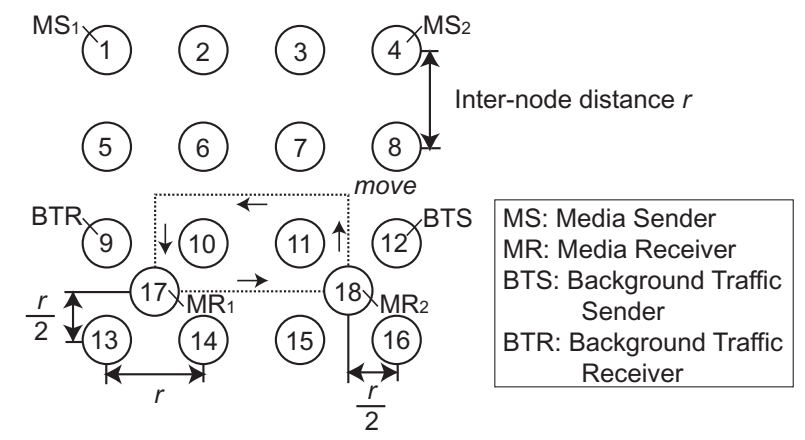

Fig. 1. Network topology.

During a TXOP, successive sequences of frame exchange are separated by SIFS (Short InterFrame Space). When a TXOP ends, the station goes into backoff. The TXOP-bursting can reduce overhead due to contention if a station has more than one MAC frame in a source buffer.

\section{Methodology of Simulation}

At first, we performed computer simulation with $n s-2$ (network simulator version 2) [15]. The network consists of 18 nodes as shown in Fig. 1. We employed OLSR (Optimized Link State Routing) as the routing protocol; it is a proactive routing protocol.

The routing protocol is one of the most important elements to construct ad hoc networks. It can affect the QoE and QoS largely. In this paper, as a first step toward the QoE assessment in mobile ad hoc networks, we have employed a primitive ad hoc routing protocol. More sophisticated routing protocols discussed in [12] and [13] can be beneficial to further enhancement of QoE; this is a future study.

The number in a circle in Fig. 1 means the node number. Nodes 1 and 4 are Media Senders (MSs), and Nodes 17 and 18 are Media Receivers (MRs). Nodes 12 and 9 are Background Traffic Sender (BTS) and Background Traffic Receiver (BTR), respectively.

Except for Nodes 17 and 18, the distance between two vertical or horizontal adjacent nodes is constant; it is referred to as the inter-node distance $r$. We employed three values of $r$ : $100 \mathrm{~m}, 120 \mathrm{~m}$, and $140 \mathrm{~m}$. Nodes 17 and 18 move along the dotted rectangle depicted in Fig. 1. In order to assess the influence of the node mobility, the movement speed of each node was set to $6 r / 120 \mathrm{~m} / \mathrm{s}$.

In the simulation, a node can receive a MAC frame when the distance between the node and the sender is smaller than $170 \mathrm{~m}$.

We utilized the freespace model of $n s-2$ as the propagation model. In ns-2, the signal strength of a MAC frame can be calculated by the propagation model and distance between the transmitter and the receiver. In calculating SNR, we assumed receiver noise strength based on Orinoco $802.11 \mathrm{~b}$ card. We also used empirical curves of BER versus SNR provided by Intersil wireless LAN chipset to obtain BER [16].

In Fig. 1, we assume Nodes 1 and 4 as the audio and video sources. Nodes 1 and 4 transmitted the media streams to Nodes 17 and 18, respectively, with UDP. We refer to the transmission
TABLE I

SPECIFICATIONS OF THE AUDIO AND VIDEO.

\begin{tabular}{|c|c|c|}
\hline & audio & video \\
\hline \hline coding method & $\begin{array}{c}\text { ITU-T G.711 } \\
\mu-l a w\end{array}$ & $\begin{array}{c}\text { MPEG1 } \\
\text { GOP I }\end{array}$ \\
\hline image size [pixel] & - & $320 \times 240$ \\
\hline average MU size [byte] & 400 & 2500 \\
\hline average MU rate [MU/s] & \multicolumn{2}{|c|}{20} \\
\hline average MU interval [ms] & 64 & 400 \\
\hline average bit rate [kb/s] & \multicolumn{2}{|c|}{120} \\
\hline duration [s] & \multicolumn{2}{|c|}{} \\
\hline
\end{tabular}

TABLE II

PARAMETERS OF THE EDCA.

\begin{tabular}{|c|c|c|c|c|c|}
\hline & $\mathrm{AC}$ & $C W_{\min }$ & $C W_{\max }$ & $\begin{array}{c}\text { AIFS } \\
{[\mu \mathrm{s}]}\end{array}$ & $\begin{array}{c}\text { TXOPLimit } \\
{[\mathrm{ms}]}\end{array}$ \\
\hline \hline audio & 3 & 7 & 15 & 50 & 3.264 \\
\hline video & 2 & 15 & 31 & 50 & 6.016 \\
\hline data & 1 & 31 & 1023 & 70 & 0 \\
\hline
\end{tabular}

unit at the application-level as a Media Unit $(M U)$; we define a video frame as a video MU and a constant number of audio samples as an audio MU. We used an audio stream of ITU-T G.711 $\mu$-law and an MPEG1 video stream, which has been prepared by encoding a part of a Japanese music clip. Table I shows the specifications of the audio and video.

Nodes 12 and 9 are used to handle an independent interference traffic flow for the media streams. Node 12 generated fixed-size IP datagrams of 1500 bytes each at exponentially distributed intervals and then sent to Node 9. We set the average amount of the interference traffic to $100 \mathrm{~kb} / \mathrm{s}$ in the simulation.

We assessed the QoE of DCF, EDCA, and TXOP. DCF is the original DCF scheme in the IEEE 802.11 standard. EDCA means the IEEE 802.11e EDCA without TXOP-bursting. TXOP is the scheme which combines the IEEE 802.11e EDCA and TXOP-bursting.

Table II shows parameter values of the EDCA in the simulation. These are default EDCA parameter values in [2]. In this table, the value of TXOPLimit for data is 0 ; this means that TXOP-bursting is not employed for the data transmission. In addition, we have assigned the same priority to the control information packets for the routing protocol as that for the audio transmission.

\section{Methodology of QoE Assessment}

In this paper, we assess QoE of the output audio-video stream by a subjective experiment. It was conducted as follows.

For subjective assessment, we made stimuli, which are objects to be evaluated, by actually outputting the audio and video MUs with the output timing obtained from the simulation. Each stimulus lasts 120 seconds.

We put the stimuli in a random order and presented them to 16 assessors, using a laptop PC with headphones. The assessors are male and female in their twenties.

A subjective score is measured by the rating-scale method, in which assessors classify each stimulus into one of a certain 
number of categories. We adopt the following five categories of impairment: "imperceptible" assigned integer 5, "perceptible, but not annoying" 4, "slightly annoying" 3, "annoying" 2 , and "very annoying" 1 . The integer value is regarded as the subjective score.

In audio-video streaming in mobile ad hoc networks, its quality can fluctuate quite largely. In the rating-scale method, each assessor is supposed to give a subjective score for a stimulus. However, it is difficult for the assessors to give the average of the perceived quality at the end of each stimulus because of the temporal fluctuation. Thus, we asked the assessors to give a score for each fragment of a stimulus as stated below.

While a stimulus is presented to each assessor, he/she classifies every instantaneous quality into one of the five categories of impairment according to his/her subjective assessment. The assessor inputs a score by the laptop PC's keyboard whenever his/her classification changes from a score that had been input immediately before. The input score is kept until the assessor changes it to another; it is sampled every second. The sampled value is assumed as the subjective score for the fragment for the second.

In this paper, we utilize the method of successive categories [17]. In order to obtain an interval scale as the QoE parameter, we first measure the frequency of each category with which the fragment of the stimulus was placed in the category by the rating-scale method. With the law of categorical judgment, we can translate the frequency obtained by the rating-scale method into an interval scale. We then perform Mosteller's test, which tests the goodness of fit between the obtained interval scale and the measurement result. The interval scale of which we have confirmed the goodness of fit is referred to as the psychological scale [17].

The assessors assessed stimuli for the three schemes: DCF, EDCA, and TXOP. For each scheme, there were three stimuli, which correspond to the inter-node distances $r$ of $100 \mathrm{~m}$, $120 \mathrm{~m}$, and $140 \mathrm{~m}$. It took about 30 minutes for an assessor to finish all assessment which includes the presentation of the original audio-video stream, a stimulus for practice, and $3 \times 3=9$ stimuli.

\section{Assessment Results}

This paper discusses the quality of the audio-video stream received at Node 18. At first, Figs. 2 through 4 depict the average number of hops from Node 4 to Node 18 as a function of elapsed time. We find in these figures that the number of hops with EDCA has almost the same tendency as that with TXOP. On the other hand, DCF has a little difference from the two schemes. This is because DCF does not change the route so quickly as EDCA or TXOP owing to the queuing delay for control packets of the routing protocol; in EDCA and TXOP, high priority is given to the control packets for channel access.

\section{A. Application-Level QoS}

In this study, we also assess the application-level QoS, which is closely related to the QoE. Although we have employed the three values of $r$ in the assessment, we only show the assessment results with $r=120 \mathrm{~m}$ because of space limitations.

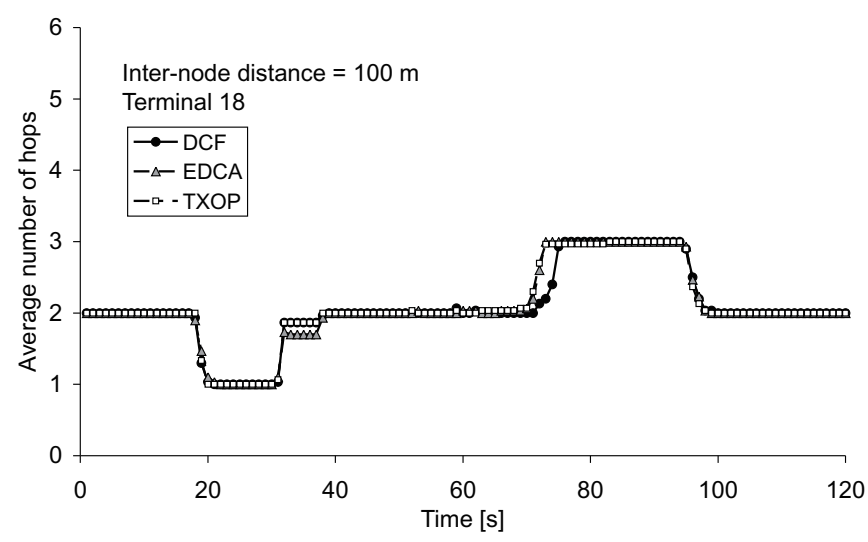

Fig. 2. Average number of hops (Inter-node distance $=100 \mathrm{~m}$ ).

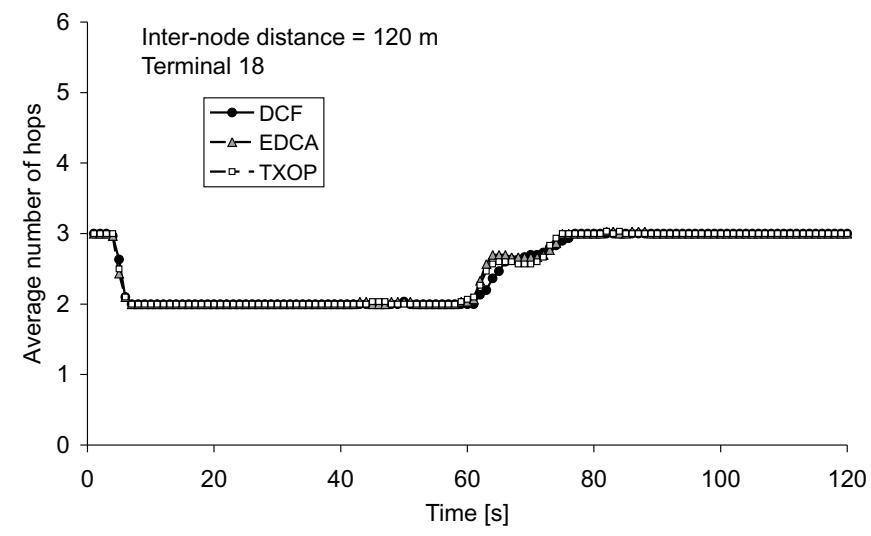

Fig. 3. Average number of hops (Inter-node distance $=120 \mathrm{~m}$ ).

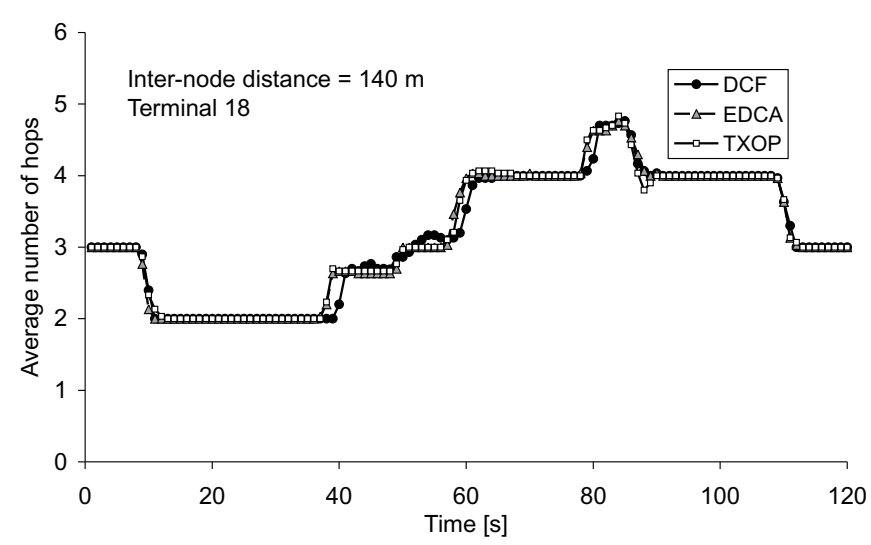

Fig. 4. Average number of hops (Inter-node distance $=140 \mathrm{~m}$ ).

The coefficient of variation of MU output interval for audio is shown in Fig. 5. It is defined as the ratio of the standard deviation of the MU output interval (i.e., the presentation time interval of two MUs at the destination) of a stream to its average and represents the smoothness of the output stream. In this figure, blanks of lines (e.g., around time 60) mean pause 


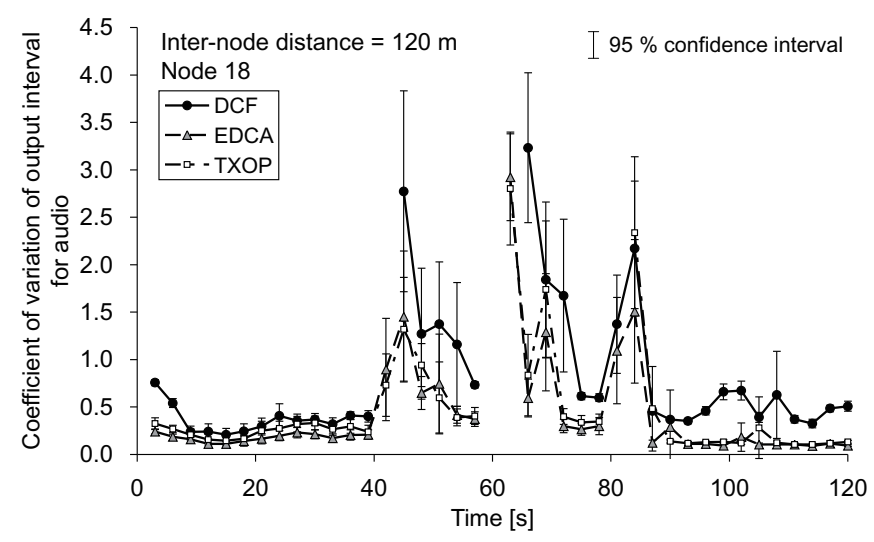

Fig. 5. Coefficient of variation of MU output interval for audio (Inter-node distance $=120 \mathrm{~m})$.

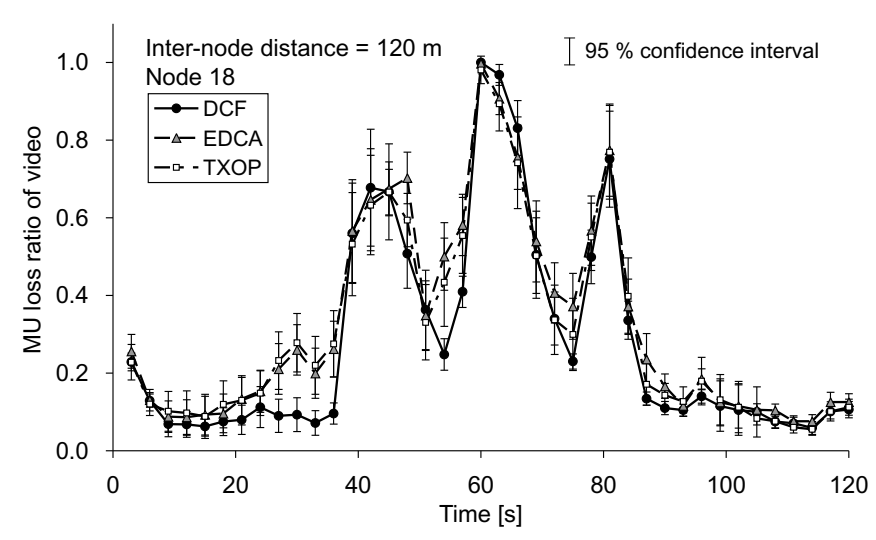

Fig. 6. MU loss ratio of video (Inter-node distance $=120 \mathrm{~m}$ ).

periods due to route disconnections.

We find in Fig. 5 that EDCA has the smallest coefficient of variation of MU output interval for audio among the three schemes. This is because the audio stream has the highest priority than the other streams in EDCA.

Figure 6 represents the MU loss ratio of video versus elapsed time. It is the ratio of the number of MUs lost to the total number of MUs transmitted from the sender terminal. In this figure, we can observe that DCF has smaller MU loss ratio of video than the other two schemes especially in the period of around time 9 to time 39. This is because EDCA and TXOP assign the smaller $\mathrm{CW}$ values for the video $\mathrm{AC}$ than DCF. When the network condition is not good, the schemes attempt much retransmission in a short period and then can reach the limit of retransmission easily.

\section{B. $Q o E$}

We calculated the QoE parameter for all the inter-node distances employed in the simulation. We processed the result in the period of time 20 through 110 . We then performed Mosteller's test, which tests the goodness of fit between the obtained interval scale and the measurement result. As a result of the test, we found that the null hypothesis that the

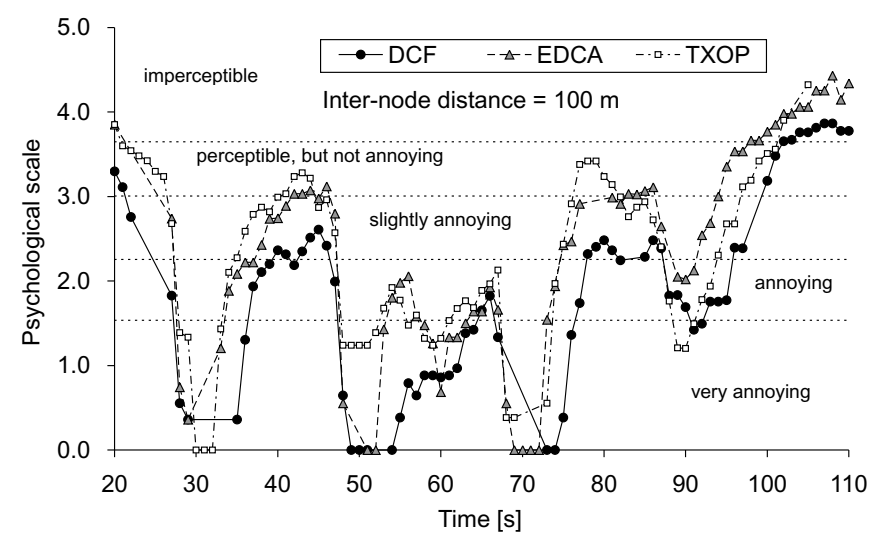

Fig. 7. Psychological scale (Inter-node distance $=100 \mathrm{~m}$ ).

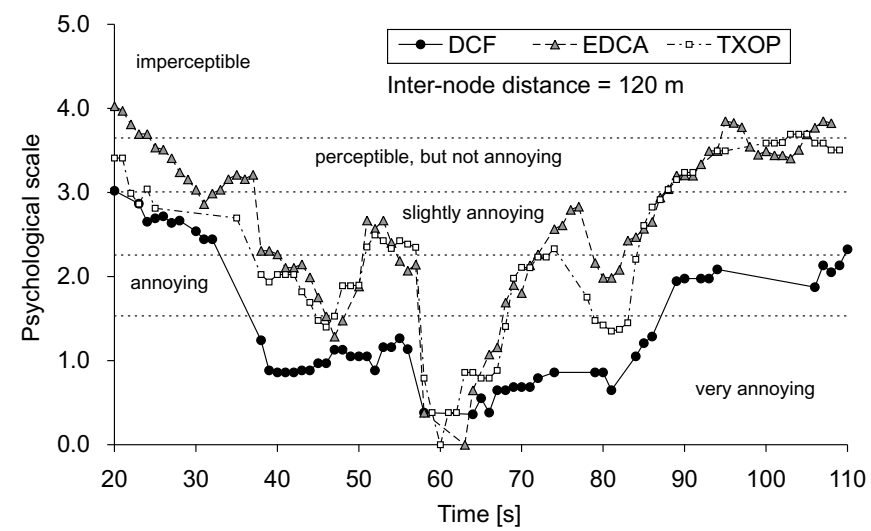

Fig. 8. Psychological scale (Inter-node distance $=120 \mathrm{~m}$ ).

obtained interval scale fits the observed data can be rejected at significance level 0.01. By removing about $24 \%$ of the fragments, we saw that the hypothesis cannot be rejected; we consider the interval scale as the psychological scale, i.e., QoE parameter.

Note that we can select any origin in the interval scale. In this paper, for convenience, we regard the minimum value of the psychological scale as the origin. Under this condition, we also calculated the lower boundaries of the categories and got 3.649 for Category 5, 3.008 for Category 4, 2.256 for Category 3 , and 1.534 for Category 2.

Figures 7, 8, and 9 plot the psychological scale versus the elapsed time for the inter-node distance $100 \mathrm{~m}, 120 \mathrm{~m}$, and $140 \mathrm{~m}$, respectively. In these figures, horizontal dotted lines show boundaries between the categories.

In Figs. 7 through 9, we notice that the values of the psychological scale are strongly affected by the route changes; the degradation of QoE occurs when the number of hops changes as we can see in Figs. 2 through 4. The routing protocol is one of the dominant factors for audio-video quality in ad hoc networks.

We find in Figs. 7 through 9 that EDCA and TXOP have higher values of the psychological scale than DCF in almost all the duration. Thus, EDCA and TXOP are also effective 


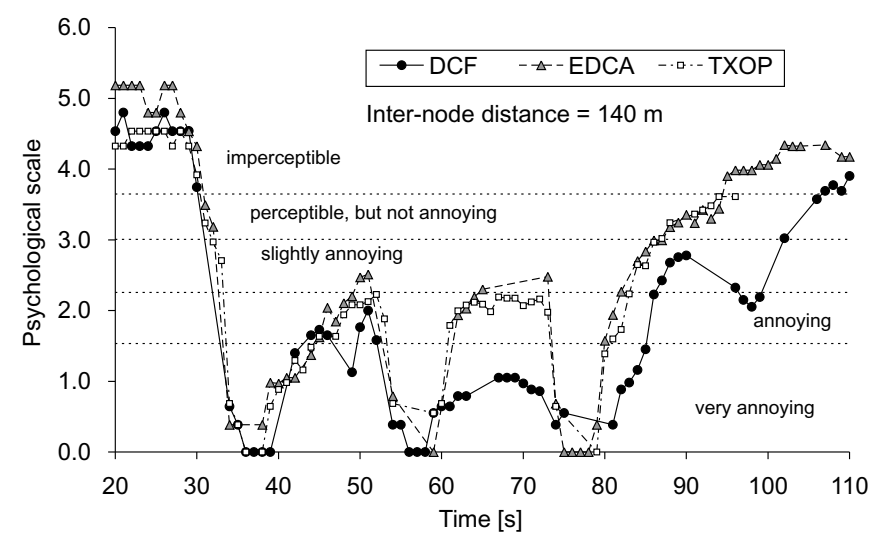

Fig. 9. Psychological scale (Inter-node distance $=140 \mathrm{~m}$ ).

in audio-video transmission over mobile ad hoc networks as well as infrastructure wireless LANs [11].

We can observe in Figs. 7 through 9 that TXOP tends to have lower QoE than EDCA. This is because we employ an audio-dominant content in the experiment, and TXOPbursting does not work effectively for the audio stream.

In Figs. 7 and 9, we see that the recovery of the psychological scale value after the route change with DCF delays against that with EDCA or TXOP. This is due to the queuing delay for control packets.

We notice in Figs. 7 through 9 that the values of the psychological scale for the inter-node distance $140 \mathrm{~m}$ for the duration from time 20 to 30 are the best among the three internode distances. This is because the route for the inter-node distance $140 \mathrm{~m}$ for this duration does not include long links, which are included in the route for the inter-node distance $100 \mathrm{~m}$ or $120 \mathrm{~m}$; the long links suffer from the channel error largely.

From the above observation, as for the application-level QoS, EDCA has good audio quality owing to the prioritized transmission, and DCF has good video quality because of its large CW size. However, from the QoE assessment results, perceived quality of audio-video with DCF is not better than that with either of the other two schemes. One of the reasons is that we have employed the music clip as the audio and video sources, and then the audio affects subjective quality more largely than the video. We should assess the QoE with various audio-video contents; this is for future study.

\section{CONCLUSION}

This paper assessed QoE and application-level QoS of audio-video transmission over mobile ad hoc networks with IEEE 802.11e EDCA. As a result, we found that EDCA and TXOP-bursting improve the QoE of audio-video streams even in mobile ad hoc networks. These schemes can achieve better application-level QoS for audio than DCF and can recover route disconnections faster.

As future work, we have to investigate the effect of crosslayering between the routing protocols and EDCA on QoE because the routing protocol affects QoE largely. We also need to assess the effect of parameter values for EDCA on QoE.

\section{ACKNOWLEDGMENT}

The authors thank Tsuyoshi Saito for his assistance in the simulation. This work was supported by the Grant-In-Aid for Scientific Research of Japan Society for the Promotion of Science under Grant 21360183.

\section{REFERENCES}

[1] Rec. ITU-T G.100/P.10 Amendment 1, "New Appendix I Definition of Quality of Experience (QoE)", Jan. 2007.

[2] "Amendment to Standard 802.11: Medium access control (MAC) enhancements for quality of service (QoS)," IEEE 802.11e Std., Sept, 2005.

[3] V. C. Frias, G. D. Delgado and M. A. Igartua, "Multipath routing for video-streaming services over IEEE 802.11e ad hoc networks," Proc. SoftCOM 2006, Sept./Oct. 2006.

[4] L. Romdhani and C. Bonnet, "A cross-layer on-demand routing protocol for delay-sensitive applications," Proc. IEEE PIMRC 2005, Sept. 2005.

[5] T. -H. Lee, A. Marshall and B. Zhou, "A QoS-based rate adaptation strategy for IEEE a/b/g PHY schemes using IEEE 802.11e in ad-hoc networks," Proc. ICNS'06, July 2006.

[6] A. Iera, A. Molinaro, G. Ruggeri and D. Tripodi, "Improving QoS and throughput in single- and multihop WLANs through dynamic traffic prioritization," IEEE Network, vol. 19, no. 4, pp. 35-44, July/Aug. 2005.

[7] A. Iera, A. Molinaro, S. Polito and G. Ruggri, "Coordinated multihop scheduling in IEEE 802.11e wireless ad hoc networks," Proc. IEEE PIMRC 2006, Sept. 2006.

[8] T. B. Reddy, J. P. John and C. S. R. Murthy, "Providing MAC QoS for multimedia traffic in 802.11e based multi-hop ad hoc wireless networks," Computer Networks, 51 (2007) 153-176.

[9] H. Zhu, G. Cao, A. Yener and A. D. Mathias, "EDCF-DM: A novel enhanced distributed coordination function for wireless ad hoc networks," Conf. Rec. IEEE ICC 2004, June 2004.

[10] C. T. Calafate, J. -C. Cano, P. Manzoni and M. P. Malumbres, "A QoS architecture for MANETs supporting real-time peer-topeer multimedia applications," Proc. IEEE ISM '05, Dec. 2005.

[11] T. Suzuki, A. Noguchi and S.Tasaka, "Effect of TXOP-bursting and transmission error on application-level and user-level QoS in audio-video transmission with IEEE 802.11e EDCA," Proc. IEEE PIMRC 2006, Sept. 2006.

[12] T. Nunome, S. Tasaka and K. Nakaoka, "Application-level and user-level QoS assessment of audio-video IP transmission over cross-layer designed wireless ad hoc networks," IEICE Trans. Commun., vol. E91-B, no. 10, pp. 3205-3215, Oct. 2008.

[13] T. Nunome and S. Tasaka, "User-level QoS assessment of audio-video IP transmission over link quality-based ad hoc networks," Proc. First Ambient Networks Workshop on Mobility, Multiaccess, and Network Management (M2NM-2007), pp. 1924, Oct. 2007.

[14] Y. Ito, S. Tasaka and R. Ito, "Continuous time assessment and mapping of user-level QoS in audio-video transmission over IP networks," Proc. IASTED Intl. Conf. COMMUNICATIONS AND COMPUTER NETWORKS, pp.230-237, Oct. 2005.

[15] "The Network Simulator - ns-2", http://www.isi.edu/nsnam/ns/.

[16] W. Xiuchao, "Simulate 802.11 b channel within ns2," http://www.comp.nus.edu.sg/wuxiucha/Sim8011ChNS2.pdf, 2004.

[17] S. Tasaka and Y. Ito, "Psychometric analysis of the mutually compensatory property of multimedia QoS," Conf. Rec. IEEE ICC 2003, May. 2003. 